\title{
Different Spectral Hounsfield Unit Curve and High-Energy Virtual Monochromatic Image Characteristics of Squamous Cell Carcinoma Compared with Nonossified Thyroid Cartilage
}

R. Forghani, M. Levental, R. Gupta, S. Lam, N. Dadfar, and H.D. Curtin

\begin{abstract}
BACKGROUND AND PURPOSE: The attenuation of normal nonossified thyroid cartilage can be similar to that of head and neck squamous cell carcinoma on CT. We compared dual-energy CT spectral Hounsfield unit attenuation characteristics of nonossified thyroid cartilage with that of squamous cell carcinoma to determine the optimal virtual monochromatic image reconstruction energy levels for distinguishing tumor from normal nonossified thyroid cartilage.
\end{abstract}

MATERIALS AND METHODS: Dual-energy CT scans from 30 patients with histopathology-proved squamous cell carcinoma at different primary sites (laryngeal and nonlaryngeal) and 10 healthy patients were evaluated. Patients were scanned with a 64-section single-source scanner with fast-kilovolt (peak) switching, and scans were reconstructed at different virtual monochromatic energy levels ranging from 40 to $140 \mathrm{keV}$. Spectral attenuation curves of tumor and nonossified thyroid cartilage were quantitatively evaluated and compared. Any part of the tumor invading the cartilage, when present, was excluded from ROI analysis to avoid cross-contamination from areas where there could be a mixture of cartilage and invading tumor.

RESULTS: Normal nonossified thyroid cartilage had a characteristic, predictable spectral attenuation curve that was different from that of tumors. The greatest difference in attenuation of nonossified cartilage compared with tumor was on virtual monochromatic images of $\geq 95 \mathrm{keV}(P<.0001)$, with sharp contrast between the relatively high attenuation of nonossified cartilage compared with that of tumor.

CONCLUSIONS: Head and neck squamous cell carcinoma has significantly different attenuation on virtual monochromatic images of $\geq 95$ keV, compared with nonossified thyroid cartilage.

ABBREVIATIONS: CNR = contrast-to-noise ratio; DECT = dual-energy CT; HNSCC = head and neck squamous cell carcinoma; NOTC = nonossified thyroid cartilage; $S H U A C=$ spectral Hounsfield unit attenuation curve; $\mathrm{VMI}=$ virtual monochromatic image

$\mathrm{H}^{\mathrm{e}}$ ead and neck squamous cell carcinoma (HNSCC) is a common malignancy of the larynx and hypopharynx. Imaging plays a key role in staging of laryngeal and hypopharyngeal cancers, and accurate staging is necessary to help determine whether a patient will undergo organ-preserving treatment or laryngec-

Received October 13, 2014; accepted after revision December 14.

From the Department of Radiology (R.F., M.L., S.L.), Jewish General Hospital, McGill University, Montreal, Quebec, Canada; Department of Radiology (R.G.), Massachusetts General Hospital, and Department of Radiology (N.D., H.D.C.), Massachusetts Eye and Ear Infirmary, Harvard Medical School, Boston, Massachusetts.

Abstract previously presented at: Annual Meeting of the American Society of Head and Neck Radiology, September 10-14, 2014; Seattle, Washington.

Please address correspondence to Reza Forghani, MD, PhD, Department of Radiology, Jewish General Hospital, Room C-212.1, 3755 Cote Ste-Catherine Rd, Montreal, QC, Canada, H3T 1E2; e-mail: rforghani@jg.mcgill.ca

- Indicates open access to non-subscribers at www.ajnr.org

三 Indicates article with supplemental on-line appendix and tables.

Indicates article with supplemental on-line photo.

http://dx.doi.org/10.3174/ajnr.A4253 tomy. ${ }^{1-7}$ An important aspect of staging is to determine whether there is thyroid cartilage invasion. ${ }^{1,2,4-9} \mathrm{CT}$ is typically the first-line technique for evaluation of laryngeal cancer, but evaluation of thyroid cartilage invasion remains a challenge on CT, in large part due to variable ossification of the thyroid cartilage. Although tumor has a different appearance from both the cortex and the fat-filled medullary space of ossified cartilage, the attenuation values of tumor and nonossified thyroid cartilage (NOTC) are very similar. ${ }^{1,10,11}$ Several criteria have been evaluated and proposed to improve the accuracy of CT for detection of cartilage invasion, but none are perfect. ${ }^{1,2,11-14}$

Dual-energy CT (DECT) evaluates tissues at different x-ray energies, enabling spectral evaluation and material tissue characterization. ${ }^{15}$ With DECT, projection data are typically obtained simultaneously or near-simultaneously at 80 and 140 kilovolts (peak) $(\mathrm{kV}[\mathrm{p}]) .{ }^{16,17}$ The measured values at the different acquisition energies can then be normalized to specific combinations of 2 different reference materials, such as iodine, water, or calcium. Furthermore, with sophisticated reconstruction algorithms, the 


\begin{tabular}{lc}
\multicolumn{1}{c}{ Summary of primary HNSCC tumor sites evaluated } \\
\hline \multicolumn{1}{c}{ Primary Site } & $\begin{array}{c}\text { No. of } \\
\text { Patients }\end{array}$ \\
\hline Untreated tumors $(n=22)$ & $7^{\text {a }}$ \\
Larynx & 1 \\
Hypopharynx & 3 \\
Retromolar trigone, anterior tonsillar pillar & 5 \\
$\quad$ Oral cavity, other & 3 \\
$\quad$ Oropharynx, other & 3 \\
Sinuses, nose & \\
Recurrent or metastatic tumors $(n=8)$ & \\
HNSCC invading parotid $(n=2)$, parapharyngeal \\
$\quad$ space metastasis, oral cavity $(n=2)$, base of tongue, \\
$\quad$ cheek, neopharynx
\end{tabular}

a Three of 7 laryngeal tumors had pathologically proven thyroid cartilage invasion.

information obtained from the different acquisitions can be combined to generate image sets at different predicted energy levels (kiloelectron volt $[\mathrm{keV}]$ ), referred to as virtual monochromatic images (VMI). Depending on their elemental properties, different tissues have distinct attenuation, signal to noise, and contrast at different VMI energy levels, enabling spectral evaluation of tissue attenuation. ${ }^{16,17}$ Although currently there is only limited evidence, emerging data demonstrate advantages of DECT for head and neck imaging, with the potential for improved tumor visualization and material characterization. ${ }^{18-21}$ Kuno et $\mathrm{al}^{22}$ have also demonstrated that the addition of iodine overlay maps can increase interobserver reproducibility and improve the specificity of CT for determination of cartilage invasion without decreasing sensitivity compared with conventional CT images alone.

On contrast-enhanced CT, NOTC has relatively high intrinsic attenuation without measurable blood supply or iodine, whereas HNSCC demonstrates increased attenuation secondary to increased iodine content after administration of IV contrast. Therefore, because of their different compositions, we hypothesized that DECT virtual monochromatic images reconstructed at different energy levels may be useful in distinguishing tumor from nonossified thyroid cartilage, and in this investigation, we compared the spectral Hounsfield unit attenuation curve (SHUAC) characteristics of HNSCC with those of NOTC. The objective of our study was to evaluate the differences in spectral attenuation and determine the optimal virtual monochromatic image reconstruction energy levels for distinguishing tumor from NOTC. This study compared the enhancing part of tumor with normal nonossified thyroid cartilage. Any part of the tumor potentially invading the cartilage, when present, was excluded from quantitative ROI analysis to avoid cross-contamination from areas where there could potentially be a mixture of cartilage and invading tumor.

\section{MATERIALS AND METHODS \\ Patients}

The study was approved by the institutional review board. Thirty patients with proved HNSCC (by biopsy and/or surgery) and 10 healthy patients who had undergone DECT between June 2013 and July 2014 were retrospectively evaluated. Tumors from different primary sites were evaluated and are summarized in the Table. They included 7 patients with laryngeal carcinoma and 1 patient with hypopharyngeal carcinoma. Healthy patients were selected ad hoc from the same period (please refer to On-line Appendix for additional details on patient selection).

\section{Image Acquisition}

All patients were scanned with the same 64-section dual-energy scanner (Discovery CT750 HD; GE Healthcare, Milwaukee, Wisconsin). All examinations were acquired after administration of IV contrast. Eighty milliliters of iopamidol (Isovue 300; Bracco, Princeton, New Jersey) was injected at a rate of $2 \mathrm{~mL} / \mathrm{s}$, and the patient was scanned after a delay of 65 seconds. Scans were acquired in dual-energy rapid 80 - to $140-\mathrm{kV}(\mathrm{p})$ switching mode by using the Gemstone Spectral Imaging (GE Healthcare) protocol. These were acquired with a Gemstone Spectral Imaging preset 15, with a large scan FOV ( $\leq 50 \mathrm{~cm}), 40-\mathrm{mm}$ beam collimation, 0.6second rotation time, and 0.984:1 helical pitch. Images were reconstructed into $1.25-\mathrm{mm}$ sections in a $25-\mathrm{cm}$ display FOV and $512 \times 512$ matrix. The average CT dose index volume for the main acquisition (entire neck to the carina) was $17.3 \mathrm{mGy}$.

\section{Image Postprocessing and Analysis}

Quantitative image analysis was performed at the Advantage Workstation 4.6 (GE Healthcare). Scans were retrospectively reconstructed into different VMI energy levels ranging from 40 to $140 \mathrm{keV}$ in $5-\mathrm{keV}$ increments. Tumor and NOTC evaluation was performed by measuring mean CT attenuation (in Hounsfield unit \pm SD) within ROIs across the entire range of VMI energy levels.

First, NOTC was evaluated in healthy patients. Eighteen ROIs were placed in the NOTC in each patient, 9 on each side, to compare and confirm similarity of the 2 sides. Thereafter, normal NOTC and tumors were evaluated in 30 patients with HNSCC. In this population, the NOTC and tumor were each evaluated with 9 ROIs placed on at least 3 separate sections. For tumors, the ROIs were placed in the homogeneous-appearing enhancing part of the tumor, avoiding areas of cystic change or necrosis. For NOTC, ROIs were placed in the nonossified part of the thyroid cartilage, avoiding the area immediately adjacent to a site of ossification. In healthy patients and in patients with tumor who had primary tumors outside the larynx, ROIs were placed bilaterally within the NOTC. In patients with laryngeal cancers, ROIs were placed in either the contralateral normal NOTC, or if placed in the ipsilateral NOTC, they were placed in an area separated by at least 5 sections $(6 \mathrm{~mm})$ from any area of contact between tumor and cartilage. For evaluation of tumor in patients with laryngeal cancer, any part of the tumor adjacent to or invading the cartilage, when present, was excluded from quantitative ROI analysis to avoid cross-contamination from areas where there could potentially be a mixture of cartilage and invading tumor.

A total of 693 ROIs were evaluated. The sizes of the ROIs varied because of differences in thickness of the NOTC, tumor size, and tumor homogeneity among patients. The minimum individual ROI diameter used was $1.5 \mathrm{~mm}$ (corresponding to a sampled area per ROI of $1.77 \mathrm{~mm}^{2}$ ), and the maximum diameter used was $5.5 \mathrm{~mm}$ (corresponding to a sampled area per ROI of 23.76 $\mathrm{mm}^{2}$ ). As discussed earlier, multiple, at least 9, ROIs were used per structure, and the average ROI area evaluated per structure (normal cartilage or tumor) was $86.8 \mathrm{~mm}^{2}$ (range, 21.2-212.1 $\mathrm{mm}^{2}$ ). The ROIs were averaged for each structure. So that the results are not biased toward larger structures (eg, larger tumors), 
the average ROI for each patient's NOTC or tumor was given equal weight when pooling data from multiple patients. Care was taken to avoid overlap of the ROI with adjacent tissues or necrotic parts of tumor to avoid volume averaging. All ROIs were placed by an attending physician with fellowship training and 4 years' postfellowship experience in neuroradiology and head and neck radiology (R.F.). Additional information on the ROI analysis is provided in the On-line Appendix.

For each patient, the mean tumor and/or normal NOTC attenuation was determined on the basis of the average Hounsfield unit of the respective ROIs in that patient. Image noise was calculated as the SD in the ROI, and the average noise for each tumor or NOTC was calculated by obtaining the average SD in their respective ROIs. ${ }^{17}$ Comparisons of tumor and NOTC attenuation were performed by obtaining the average Hounsfield unit in all patients across the VMI range (40-140 keV). Tumor and NOTC contrastto-noise ratios (CNRs) were calculated individually for each patient by using the following formula: $C N R=$ (Average Tumor Attenuation - Average Cartilage Attenuation)/Square Root [Variance (Tumor) + Variance (Cartilage) $].{ }^{17}$ In 3 patients with HNSCC, the thyroid cartilage was completely ossified. In this subgroup, the CNR calculation was performed by using the average normal NOTC attenuation from the other 37 patients (27 with HNSCC and 10 healthy patients).

\section{Statistical Analysis}

Results were reported as mean \pm SD. Pooled average SHUAC curves were generated from 40 to $140 \mathrm{keV}$, in $5-\mathrm{keV}$ increments for comparison of NOTC and tumor. For comparison of means from 2 different groups, an unpaired 2-tailed $t$ test was used. For comparison of multiple $(>2)$ groups, 1-way ANOVA with the Tukey multiple comparison test was used. Variance was calculated by using the standard formula: variance $=\mathrm{SD}^{2}$. A $P$ value $<$ .05 was statistically significant. We used GraphPad Prism software, Version 6.005, for statistical analysis (GraphPad Software, San Diego, California).

\section{RESULTS}

We first evaluated the spectral Hounsfield unit characteristics of normal NOTC in a group of 10 healthy patients (Fig $1 A$ ). The NOTC attenuation was highest at $40 \mathrm{keV}$ and progressively decreased on higher kiloelectron volt VMIs (Fig 1A). There was no significant difference in the spectral Hounsfield unit characteristics of the right compared with the left NOTC. We then evaluated normal or unaffected NOTC in 30 patients with HNSCC (average age, 72 years; range, 56-97 years; 15 men, 15 women). Their primary tumor sites are summarized in the Table. Of the patients from the tumor group, 27 normal (or unaffected) NOTCs were included. In 3 patients, the entire thyroid cartilage was ossified and, therefore, was excluded from the analysis. There was no significant difference in NOTC between healthy subjects and those with normal or unaffected NOTC in the group of patients with HNSCC (Fig 1B). Subgroup analysis of normal/unaffected NOTC in the healthy patient group, patients with laryngeal cancer, those with untreated primaries outside the larynx, and those with recurrent/metastatic tumors was also performed with 1-way ANOVA with Tukey multiple comparison tests. There was no significant
Normal NOTC: spectral HU curve comparison of right and left side in the normal patient group

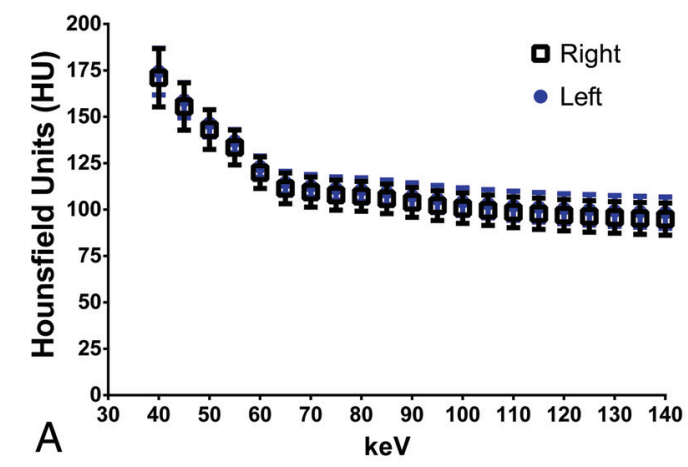

Spectral HU curve comparison of normal (or unaffected) NOTC in normal and tumor patient groups

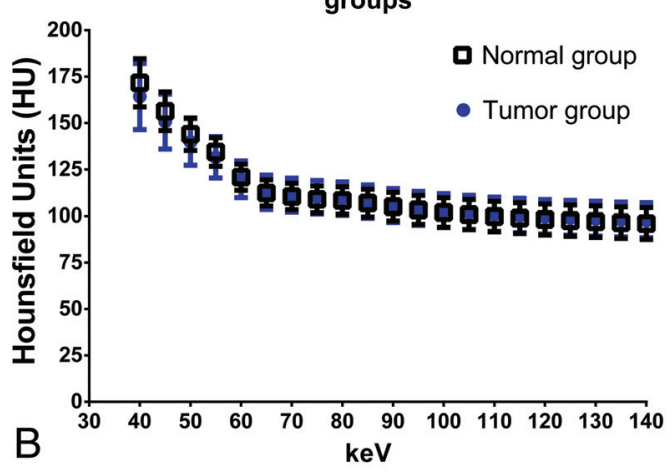

Spectral HU curve characteristics of normal NOTC: pooled normal and tumor groups

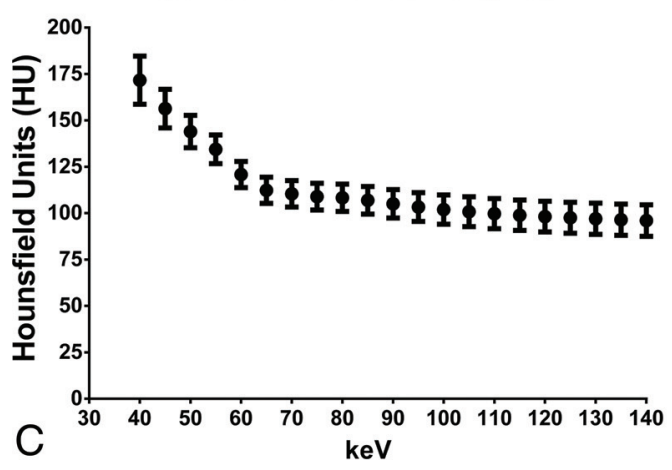

FIG 1. Comparison of spectral Hounsfield unit characteristics of normal nonossified thyroid cartilage in healthy subjects and patients with tumors. A, Baseline characteristics of NOTC evaluated in 10 healthy subjects is shown. For each patient, NOTC was evaluated with 9 ROIs on each side, corresponding to 18 ROIs total per patient, and $180 \mathrm{ROIs}$ ( 90 ROls for each side of the NOTC/curve). There was no significant difference in NOTC attenuation between the right and left sides across the virtual monochromatic range of $40-140 \mathrm{keV}$. B, Comparison of normal NOTC in healthy patients (right and left combined; $n=10$, by using 180 ROIs) and normal or unaffected NOTC in the tumor group ( $n=27$, by using 243 ROIs). C, Pooled spectral Hounsfield unit curve demonstrating characteristics of normal NOTC in the entire patient population $(n=37)$. Values shown are mean $\pm \mathrm{SD}$.

difference in the attenuation of normal NOTC between any of the groups, at any of the kiloelectron volts evaluated between 40 and $140 \mathrm{keV}$. The normal and unaffected NOTC spectral Hounsfield unit attenuation measurements from healthy patients and those 
with tumor were combined and pooled from hereon for other comparative analyses (Fig 1C).

We next evaluated and compared the spectral attenuation characteristics of HNSCC and NOTC. Untreated primaries from the larynx $(n=7)$, extralaryngeal sites $(n=15)$, and recurrent/

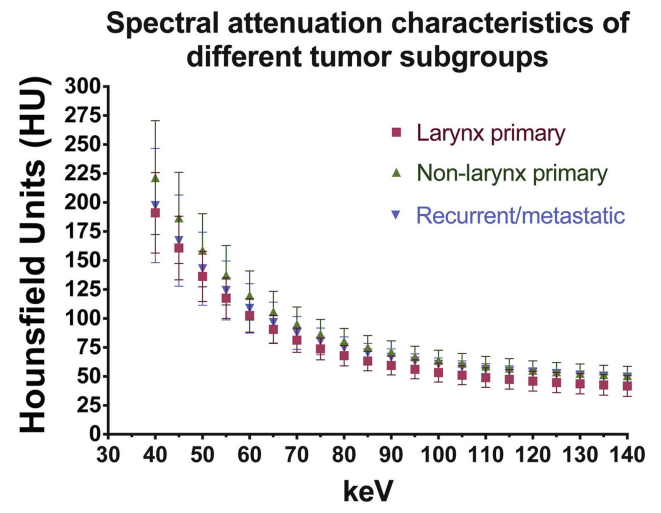

FIG 2. Comparison of spectral Hounsfield unit characteristics of different tumor subgroups: untreated larynx primaries $(n=7)$, untreated nonlarynx primaries $(n=15)$, and recurrent/metastatic tumors $(n=8)$. Note that the spectral Hounsfield unit attenuation curves of all the HNSCC tumor groups follow a similar trend, with increased attenuation of enhancing tumor at lower kiloelectron volts and decreased attenuation at higher kiloelectron volts. Values shown are mean \pm SD.

Spectral HU curves of HNSCC and normal NOTC

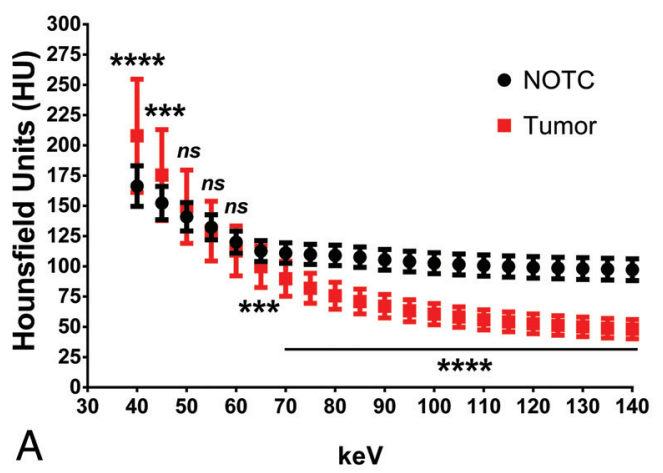

Spectral HNSCC-NOTC contrast to noise (CNR)

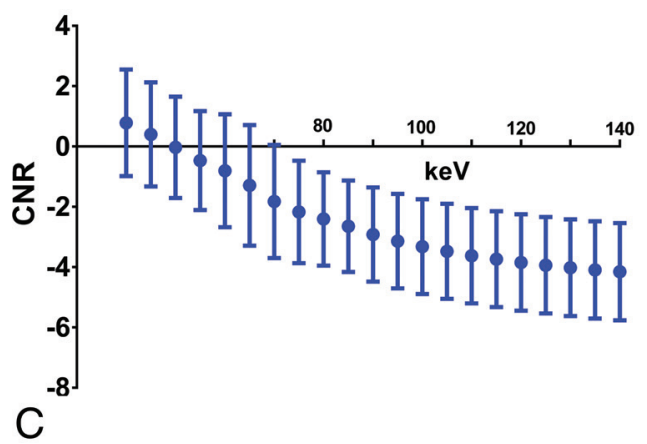

metastatic tumors $(n=8)$ were evaluated (Fig 2). All tumor subgroups had overall similar SHUAC characteristics (Fig 2). As expected, the attenuation of tumors was highest at 40-keV VMI, the kiloelectron volt closest to the k-edge of iodine. Given the similarity in their SHAUCs, the tumors were all combined and compared with NOTC (Fig $3 A$ ). The SHUAC of tumors differed from that of NOTC, with a statistically significant difference in the mean attenuation of all tumors combined compared with NOTC at all kiloelectron volts except 50, 55, and $60 \mathrm{keV}$ (Fig $3 A, P<$ $.001)$.

At $70 \mathrm{keV}$, the VMI energy level typically considered to be similar to that obtained with conventional single-energy CT, ${ }^{23}$ there was a small difference between mean tumor and NOTC attenuation, with overlap between the 2 groups on the scatterplot (Fig $3 B$ ). At the lowest extreme of the curve, at $40 \mathrm{keV}$, both tumor and NOTC had the highest average attenuation. However, the difference between tumor and NOTC was best seen on the higher kiloelectron volt VMIs, due to a combination of differences in attenuation and reduced variation (SD) of the measured values in different patients (Fig $3 A,-B$ ). Indeed, in the groups evaluated in this study, there was no overlap between individual mean tumor and NOTC attenuation on VMIs reconstructed at $\geq 95$ $\operatorname{keV}($ Fig $3 B)$.

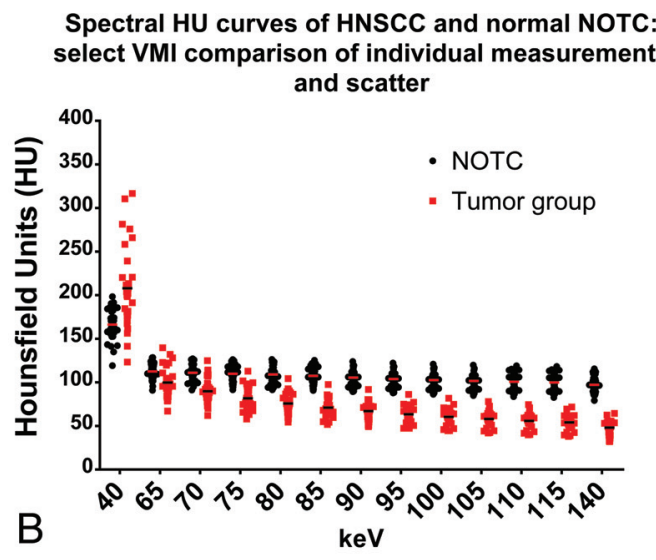

Spectral HU curves of HNSCC and normal NOTC: subgroup analysis

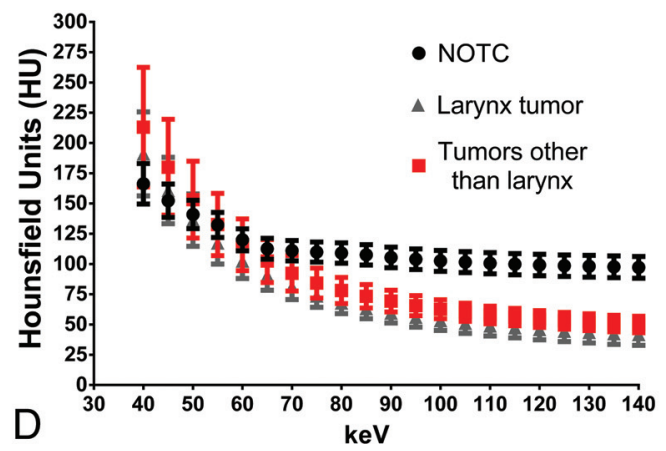

FIG 3. Spectral Hounsfield unit characteristics of head and neck squamous cell carcinoma compared with normal nonossified thyroid cartilage. $A$, Spectral Hounsfield unit curve of HNSCC at different primary sites ( $n=30$ ) compared with normal (or unaffected) NOTC ( $n=37 ; 27$ normal/unaffected NOTCs from the patients with tumor group and 10 NOTCs from healthy patients). B, Scatterplot of individual tumor densities at selected VMIs shows overlap between tumor and NOTC densities at many VMls but complete separation at high-energy VMls. C, Contrast-to-noise analysis performed at different kiloelectron volt VMIs demonstrating the higher absolute CNR at high-energy VMIs. $D$, Subgroup analysis of the laryngeal $(n=7)$ and nonlaryngeal (including a case of hypopharyngeal tumor, $n=23)$ primary sites. Three asterisks denotes $P<.001 ; 4$ asterisks denotes $P<.0001$; and ns, not significantly different. 

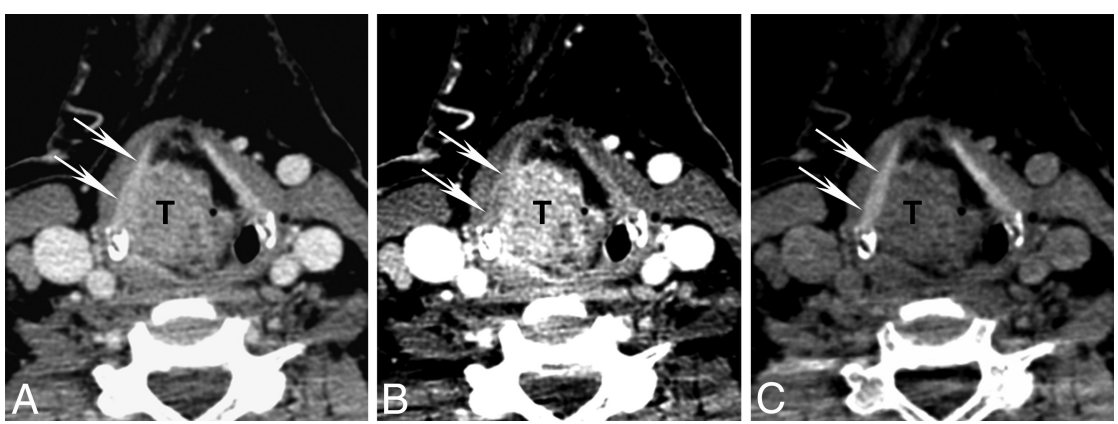

FIG 4. Differences in attenuation of NOTC (arrows) and tumor (T) on different kiloelectron volt VMls. Axial contrast-enhanced CT images are shown from a patient with a supraglottic squamous cell carcinoma with the same section reconstructed as a virtual monochromatic images at 70, 40, and $140 \mathrm{keV}$. A, Seventy-kiloelectron volt VMI shows tumor abutting the inner surface of the right NOTC. $B$, Forty-kiloelectron volt VMI shows greater attenuation of the tumor than on the 70-keV VMI due to iodine content, increased contrast between tumor and NOTC, and somewhat better depiction of tumor-NOTC interface. $C$, One hundred forty-kiloelectron volt VMI shows suppression of tumor attenuation but preserved high attenuation in the NOTC. Note the qualitative contrast improvement with clear distinction of tumor-NOTC interface.
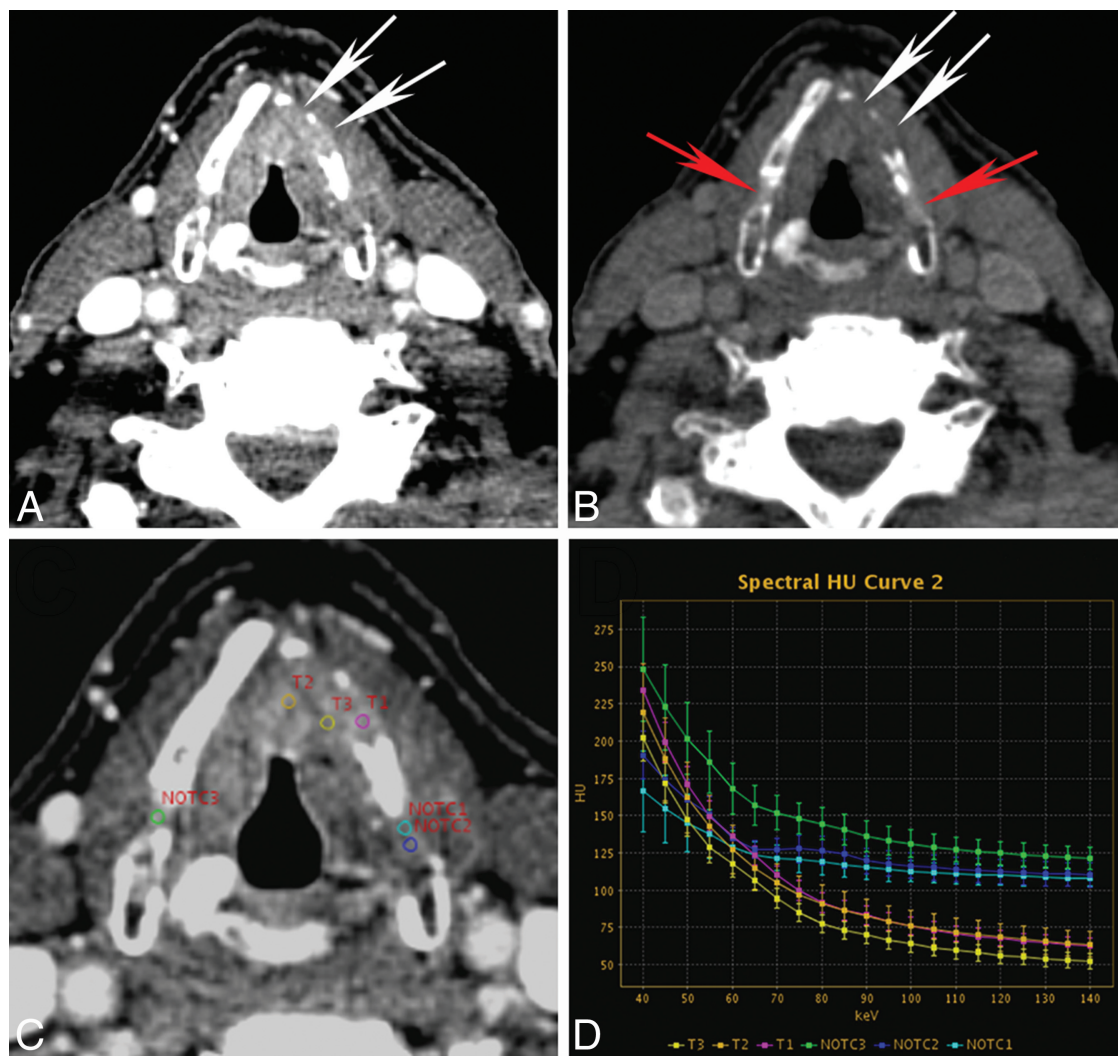

FIG 5. Quantitative spectral Hounsfield unit attenuation characterization of tumor and NOTC in a patient with pathologically proved tumor invasion into the thyroid cartilage. VMIs reconstructed at $40 \mathrm{keV}(A)$ and $140 \mathrm{keV}(B)$ demonstrate tumor invasion into the anterior left thyroid cartilage (white arrows). Note variable ossification of the left thyroid cartilage and minimal focal nonossification of the right thyroid cartilage. On the 140-keV VMI, attenuation due to invasion of the left thyroid cartilage (white arrows) is suppressed, appearing as a defect, compared with the normal ipsilateral NOTC and contralateral NOTC (red arrows). C and D, An example of quantitative analysis is shown, demonstrating individual spectral Hounsfield unit attenuation curves for different ROls. Note the differences in spectral attenuation characteristics of tumor (T) compared with normal ipsilateral and contralateral NOTC. The spectral curve shows clear attenuation separation in the higher kiloelectron volt range. Please note that the curves shown in this figure are those for individual ROIs. In earlier figures, the average from multiple ROls (at least 9) per structure, per patient, was used to calculate pooled mean attenuation for each group or subgroup (in addition, areas of invasion were not included in the earlier pooled analysis).
Subgroup analysis of the laryngeal $(n=7)$ primaries demonstrated similar trends in SHUAC, with persistent clear separation from the normal NOTC curve in the high kiloelectron volt range (Fig 3D). Subgroup analysis comparing NOTC with the untreated laryngeal primaries, untreated primaries outside the larynx, and recurrent/metastatic tumors also demonstrated a statistically significant difference between NOTC and all tumor subgroups on VMIs reconstructed at $\geq 70 \mathrm{KeV}(P<.0001,1$-way ANOVA), with the greatest difference at of $\geq 95 \mathrm{KeV}$.

We also calculated the CNR to factor in differences in noise levels at different VMI energy levels. As in the previous observations, the absolute CNR was highest on high kiloelectron volt images (Fig $3 C$ ). Note that the negative values in Fig $3 C$ indicate that tumor has a lower attenuation than NOTC at these kiloelectron volts. Therefore, tumor invasion of NOTC would be expected to appear as a low-attenuation defect in the otherwise high-attenuation NOTC (Figs 4 and 5).

\section{DISCUSSION}

Accurate determination of thyroid cartilage invasion is important for proper staging and treatment planning of laryngeal and hypopharyngeal cancers. ${ }^{1-8}$ Evaluation of thyroid cartilage invasion on imaging remains a challenge because of variable ossification and appearance of thyroid cartilage and similarities in attenuation of tumor and NOTC on CT. ${ }^{1,2,10,11}$ While tumor preferentially invades the ossified part of the cartilage, the similarity in appearance of NOTC and tumor can make definitive assessment for invasion challenging in cases in which tumor abuts the nonossified part of thyroid cartilage. Clear distinction of invasive tumor and focal nonossification of the cartilage may not be possible.

Kuno et $\mathrm{al}^{22}$ demonstrated that DECT iodine overlay images improved the specificity of detection of laryngeal cartilage invasion. In our investigation, we compared the spectral Hounsfield unit characteristics of tumor and NOTC by using a single-source DECT with rapid kiloelectron volt switching to determine the utility of VMIs reconstructed at var- 
ious kiloelectron volt levels for distinguishing tumor from NOTC. Our study expands on observations by Kuno et al that DECT can be helpful in the evaluation of laryngeal cartilage in patients with HNSCC. In our study, we evaluated another aspect of DECT, VMIs reconstructed at different kiloelectron volt energy levels, to characterize and compare HNSCC and unaffected NOTC.

Although in this investigation we did not directly evaluate thyroid cartilage invasion, the observations in this study are a step toward application of VMIs and SHUAC analysis for assessment of NOTC and proceeding to studies evaluating NOTC invasion. We demonstrate that HNSCC has a very different spectral Hounsfield unit attenuation curve than normal NOTC. Although there was some difference in mean attenuation between NOTC and tumor at $70 \mathrm{keV}$, the VMI energy level similar to that of a standard single-energy CT acquisition, ${ }^{23}$ the difference was small and there was overlap between individual tumor and NOTC attenuation. This finding reflects the challenges that can be encountered in distinguishing NOTC from tumor on conventional CT.

On the other hand, there were significant differences in attenuation at either extreme of the VMI energy curve, with the distinction between tumor and NOTC best achieved on VMIs of $\geq 95 \mathrm{keV}$. On high kiloelectron volt VMIs, iodine attenuation in enhancing tumor is partially suppressed, whereas the normal NOTC retains a relatively high and fairly uniform attenuation and would therefore be distinct from adjacent tumor (Fig 4). Against the high-attenuation background of NOTC, tumor would be expected to appear as a relatively low-attenuation defect that stands out from the normal thyroid cartilage (Fig 5). Normal NOTC has uniform attenuation without gaps or defects. ${ }^{24}$ On the basis of the results of our study, one would expect that VMIs may be used qualitatively to assess NOTC integrity (Figs 4 and 5). In cases where there is ambiguity, supplemental evaluation with quantitative ROI analysis and SHUAC analysis may also be helpful. A potential example of such application, demonstrating individual ROI curves, is shown in Fig 5. On the basis of the observations in this study, we believe that future investigations directly evaluating applications of high-energy VMI and SHUAC for thyroid cartilage invasion are warranted. Because many patients without thyroid cartilage invasion or with minor invasion may not be treated surgically, such studies will be designed to correlate imaging findings with long-term patient outcome rather than on the basis of histopathologic correlation alone.

Compared with iodine overlay maps, high kiloelectron voltreconstructed VMIs are similar to standard sequences to which radiologists are accustomed, possibly providing an advantage for implementation and user acceptance. In addition, high kiloelectron volt VMIs can result in decreased artifacts associated with metallic implants and other materials. ${ }^{25,26}$ At this time, both high-energy kiloelectron volt VMIs and iodine overlay maps are likely to be useful for increasing the accuracy of CT for determination of laryngeal cartilage invasion, and a comparison of the 2 and evaluation of potential complementary application in thyroid cartilage assessment is an interesting topic for future research.

Because this work is a general characterization of HNSCC, we used both primary laryngeal tumors and tumors outside the larynx. In addition, we evaluated recurrent/metastatic tumors. Although there were small differences in mean attenuation among the different tumor subgroups, all of the tumor groups had similar spectral attenuation characteristics (Fig 2). Furthermore, similar differences in NOTC and tumor attenuation with the greatest difference on high kiloelectron volt VMIs were observed when NOTC was compared with either the entire group of 30 tumors and specific subgroups.

Limitations of this study include the retrospective design and small number of cases. In addition, there was no pathologic confirmation of uninvaded NOTC. Nonetheless, our study demonstrates clear qualitative and quantitative differences in spectral Hounsfield unit attenuation curves of HNSCC compared with NOTC. We believe that our investigation and that by Kuno et $\mathrm{al}^{22}$ provide the basis for future, ideally prospective, studies to evaluate the advantages of DECT, including high kiloelectron volt VMIs, for detecting thyroid cartilage invasion and predicting patient outcome.

\section{CONCLUSIONS}

HNSCC has different spectral Hounsfield unit attenuation characteristics with significantly different attenuation on VMIs of $\geq 95$ $\mathrm{keV}$ compared with nonossified thyroid cartilage. Therefore, DECT has the potential to improve accuracy for distinguishing tumor and NOTC and to improve evaluation of cartilage invasion by laryngeal cancer.

\section{ACKNOWLEDGMENTS}

We thank Veronika Glyudza for her assistance in data preparation and processing.

Disclosures: Reza Forghani-UNRELATED: Consultancy: Biogen Idec, Collège des Médecins du Québec, Comments: no relation to submitted work; one time honorarium for the "Neuroradiology Scientific Exchange Program: Focus on the Role of MRI in the Detection, Diagnosis and Monitoring of Natalizumab-Associated Progressive Multifocal Leukoencephalopathy in Patients with Multiple Sclerosis," Montreal, Quebec, Canada, February 22, 2014; expert consultant for Collège des Médecins du Québec (Telemedicine Committee, quality assurance); Expert Testimony: Collège des Médecins du Québec, Comments: quality assurance reviews (no relation to current work); Payment for Lectures (including service on Speakers Bureaus): no relation to submitted work; "Peer Review for Departmental QA and Audits: Essentials of a Successful Program, Benchmarks for Evaluation, and Challenges," Ontario Association of Radiologists Continuing Medical Education Peer Review Course, Ontario Bar Association, Toronto, Ontario, Canada, June 7, 2014; "Sinus Anatomy: Normal and Postoperative," Ontario Association of Radiologists Continuing Medical Education Head and Neck Imaging Course, Part I, Ontario Bar Association, Toronto, Ontario, Canada, May 24, 2014; Visiting Professor, McMaster University Health Sciences Center; Radiology Grand Rounds, St. Joseph's Healthcare Hamilton ("Applications of Diffusion-Weighted MR Imaging in the Evaluation of Acute Ischemia and Non-Ischemic Neurological Disorders"), Hamilton, Ontario, Canada, January 22, 2013; Stock/ Stock Options: Real Time Medical Inc, Comments: no relation to submitted work; member, Medical and Quality Assurance Committee. Mark Levental-UNRELATED: Consultancy: Biogen Idec; Payment for Lectures (including service on Speakers Bureaus): Biogen Idec, Comments: member of speakers bureau. Rajiv Gupta-UNRELATED: Board Membership: Actelion, Comments: advising the company on the use of imaging in their drug trials; Grants/Grants Pending: Siemens, ${ }^{*}$ Comments: research grant to the institution; Travel/Accommodations/Meeting Expenses Unrelated to Activities Listed: Siemens, Comments: travel support for a symposium organized by Siemens. Hugh D. Curtin—UNRELATED: Royalties: Elsevier, Comments: royalties for textbook, not related to this article. *Money paid to the institution.

\section{REFERENCES}

1. Hermans R. Staging of laryngeal and hypopharyngeal cancer: value of imaging studies. Eur Radiol 2006;16:2386-400

2. Kuno H, Onaya H, Fujii S, et al. Primary staging of laryngeal and hypopharyngeal cancer: CT, MR imaging and dual-energy CT. Eur J Radiol 2014;83:e23-35 
3. Ferlito A, Silver CE, Howard DJ, et al. The role of partial laryngeal resection in current management of laryngeal cancer: a collective review. Acta Otolaryngol 2000;120:456-65

4. Hartl DM, Landry G, Hans S, et al. Organ preservation surgery for laryngeal squamous cell carcinoma: low incidence of thyroid cartilage invasion. Laryngoscope 2010;120:1173-76

5. Jenckel F, Knecht R. State of the art in the treatment of laryngeal cancer. Anticancer Res 2013;33:4701-10

6. Knab BR, Salama JK, Solanki A, et al. Functional organ preservation with definitive chemoradiotherapy for $\mathrm{T} 4$ laryngeal squamous cell carcinoma. Ann Oncol 2008;19:1650-54

7. Lefebvre JL. Larynx preservation. Curr Opin Oncol 2012;24:218-22

8. Edge SB, Byrd DR, Compton CC, et al. AJCC Cancer Staging Manual. New York: Springer-Verlag; 2010

9. Becker M, Zbaren P, Delavelle J, et al. Neoplastic invasion of the laryngeal cartilage: reassessment of criteria for diagnosis at CT. $R a$ diology 1997;203:521-32

10. Archer CR, Yeager VL. Evaluation of laryngeal cartilages by computed tomography. J Comput Assist Tomogr 1979;3:604-11

11. Mafee MF, Schild JA, Michael AS, et al. Cartilage involvement in laryngeal carcinoma: correlation of $\mathrm{CT}$ and pathologic macrosection studies. J Comput Assist Tomogr 1984;8:969-73

12. Becker M. Neoplastic invasion of laryngeal cartilage: radiologic diagnosis and therapeutic implications. Eur J Radiol 2000;33:216-29

13. Beitler JJ, Muller S, Grist WJ, et al. Prognostic accuracy of computed tomography findings for patients with laryngeal cancer undergoing laryngectomy. J Clin Oncol 2010;28:2318-22

14. Zbäre P, Becker M, Lang H. Pretherapeutic staging of laryngeal carcinoma: clinical findings, computed tomography, and magnetic resonance imaging compared with histopathology. Cancer 1996;77: 1263-73

15. Johnson TR, Fink C, Schönberg SO, et al, eds. Dual Energy CT in Clinical Practice. Berlin: Springer-Verlag; 2011

16. Johnson TR. Dual-energy CT: general principles. AJR Am J Roentgenol 2012;199:S3-8

17. Pomerantz SR, Kamalian S, Zhang D, et al. Virtual monochromatic reconstruction of dual-energy unenhanced head CT at $65-75 \mathrm{keV}$ maximizes image quality compared with conventional polychromatic CT. Radiology 2013;266:318-25

18. Tawfik AM, Kerl JM, Bauer RW, et al. Dual-energy CT of head and neck cancer: average weighting of low- and high-voltage acquisitions to improve lesion delineation and image quality: initial clinical experience. Invest Radiol 2012;47:306-11

19. Vogl TJ, Schulz B, Bauer RW, et al. Dual-energy CT applications in head and neck imaging. AJR Am J Roentgenol 2012;199:S34-39

20. Tawfik AM, Kerl JM, Razek AA, et al. Image quality and radiation dose of dual-energy CT of the head and neck compared with a standard 120-kVp acquisition. AJNR Am J Neuroradiol 2011;32: 1994-99

21. Srinivasan A, Parker RA, Manjunathan A, et al. Differentiation of benign and malignant neck pathologies: preliminary experience using spectral computed tomography. J Comput Assist Tomogr 2013; 37:666-72

22. Kuno $\mathrm{H}$, Onaya $\mathrm{H}$, Iwata R, et al. Evaluation of cartilage invasion by laryngeal and hypopharyngeal squamous cell carcinoma with dualenergy CT. Radiology 2012;265:488-96

23. Patel BN, Thomas JV, Lockhart ME, et al. Single-source dual-energy spectral multidetector CT of pancreatic adenocarcinoma: optimization of energy level viewing significantly increases lesion contrast. Clin Radiol 2013;68:148-54

24. Dadfar N, Seyyedi M, Forghani R, et al. Computed tomography appearance of normal nonossified thyroid cartilage: implication for tumor invasion diagnosis. J Comput Assist Tomogr 2015;39:240 - 43

25. Srinivasan A, Hoeffner E, Ibrahim M, et al. Utility of dual-energy CT virtual $\mathrm{keV}$ monochromatic series for the assessment of spinal transpedicular hardware-bone interface. AJR Am J Roentgenol 2013;201:878-83

26. Stolzmann P, Winklhofer S, Schwendener N, et al. Monoenergetic computed tomography reconstructions reduce beam hardening artifacts from dental restorations. Forensic Sci Med Pathol 2013;9: $327-32$ 